\title{
Exploración de las capacidades de la academia costarricense en Relaciones Internacionales para la creación de una estrategia de poder inteligente aplicada a la política exterior
}

\author{
Evaluation of the capabilities of the Costa Rican academy of International Relations for the \\ creation of a strategy of Intelligent Power applied to Foreign Policy
}

\author{
Luis Diego Salas Ocampo \\ Universidad Nacional, Costa Rica. luis.salas.ocampo@una.cr
}

Fabiana María Jenkins Arias

Universidad Nacional, Costa Rica. fabiejenks@gmail.com

Fecha de recepción: 23/9/2015. Fecha de aceptación: 20/5/2016

Fecha de publicación: 20/06/2016

\begin{abstract}
Resumen: Este trabajo explora la factibilidad de que la academia costarricense donde se imparte Relaciones Internacionales y el Ministerio de Relaciones Exteriores y Culto genere inputs de conocimiento que se traduzcan en outputs de política exterior en el subsistema Centroamérica. Los informantes son personal coordinador de carreras de universidades públicas y privadas, personal académico y el Señor Canciller de la República. Si se desea iniciar una acción en esta línea, debe comenzarse con el tema de medio ambiente, mejorarse los criterios de pertinencia y relevancia de los procesos de investigación académica, y dar un salto cualitativo a la investigación aplicada. Deberá administrarse, también, el hecho de que la academia costarricense en Relaciones Internacionales no tiene experiencia en la formación de think tank.
\end{abstract}

Palabras claves: Academia; sistema; Centroamérica; poder inteligente; cancillería; investigación.

\begin{abstract}
This paper explores the possibility of Costa Rican schools teaching International Relations and the Ministry of Foreign Affairs to generate knowledge input that can be translated into external policy knowledge output in the Central American subsystem. Informants included program coordinators and professors from public and private universities and the Minister of Foreign Affairs. In order to implement an action in this area, the following should be taken into consideration: first consider environmental sustainability, improve relevance criteria of academic research processes, and improve the quality of applied research. In addition, it should be considered that Costa Rican schools that teach International Affairs do not have experience teaching Think Tanks.
\end{abstract}

Keywords: Educational Institutions; System; Central America; Smart Power; Ministry of Foreign Affairs; Research 


\section{Introducción}

El proyecto Fortalecimiento de las capacidades de investigación mediante el uso de software cuantitativo, cualitativo y simuladores de negocios (FOCAIS), de la Escuela de Relaciones Internacionales de la Universidad Nacional de Costa Rica, se ha planteado, como uno de los ejes de acción, la incorporación de pensamiento joven en temas estratégicos del país en los campos de su interés. Producto de ello, este documento analiza la importancia de la Gestión del Conocimiento (GC) como herramienta estratégica de los Estados para la acción en el campo de la política exterior y su proyección en el sistema internacional.

Se analiza el caso de Costa Rica. Se trabaja partiendo de la necesidad de un accionar coordinado entre el ente rector de la política exterior (Ministerio de Relaciones Exteriores y Culto) y la academia costarricense en una disciplina concreta, las Relaciones Internacionales. Se identifica que tal sinergia puede ser potencialmente una plataforma de smart power para que el país se proyecte como líder en creación de conocimiento académico (en Centroamérica) sobre campos específicos que le pueden generar mayores cuotas de poder en lo diplomático y lo económico; y cuyas áreas impliquen un mayor desarrollo del país.

Se sostiene que Costa Rica puede lograr un mejor posicionamiento en el sistema internacional utilizando la calidad de su educación superior como una fortaleza que, unida a una administración del conocimiento, manejo del capital humano y estructural, desde una perspectiva estratégica en términos de política exterior, puede incidir en un aumento del protagonismo tanto de la disciplina de las Relaciones Internacionales a lo interno del país, así como de Costa Rica en el escenario regional en términos de sus temas medulares de política exterior. Al menos teóricamente, resulta posible que ante el escenario de que la academia de Relaciones Internacionales costarricense tome protagonismo en las decisiones internacionales del gobierno, la política exterior del país logrará un grado mayor de profesionalización y, con esto, la oportunidad de crear estrategias asertivas que posicionen a Costa Rica como un referente en ciertas temáticas de conveniencia para la proyección de sus intereses.

Este trabajo se desarrolla desde la teoría de sistemas. Costa Rica se comprende, en su accionar internacional, como una sinergia de procesos que interaccionan en movimientos de cambio e innovación. De esto, se comprende la necesidad de retroalimentación de las estrategias de política exterior, a fin de que estas puedan ajustarse de acuerdo con los niveles de competencia y cambio en la estructura de poder en el sistema internacional. Las estrategias de política exterior costarricense son abordadas como outputs (salidas) específicos que salen al interactuar con el sistema o subsistema internacional. Este último, es la región centroamericana comprendida como un subsistema en que el proceso (Costa Rica) está inmerso.

En la región, los outputs interactúan continuamente con sus agentes (representantes nacionales, universidades, individuos, grupos de poder, etc.) generando procesos de retroalimentación 
y construcción de conocimientos que son comprendidos como nuevos insumos o inputs (entradas) que le acarrean cambios, necesidades, problemas o beneficios al proceso (Costa Rica). Si la estrategia de política exterior ha sido bien gestionada, y la elaboración de los outputs responde a las necesidades del país y son capaces de interactuar positivamente en el sistema, entonces los inputs deberán ser beneficiosos. En la figura 1 se entiende el mecanismo de funcionamiento de inputs y outputs en el subsistema Centroamérica.

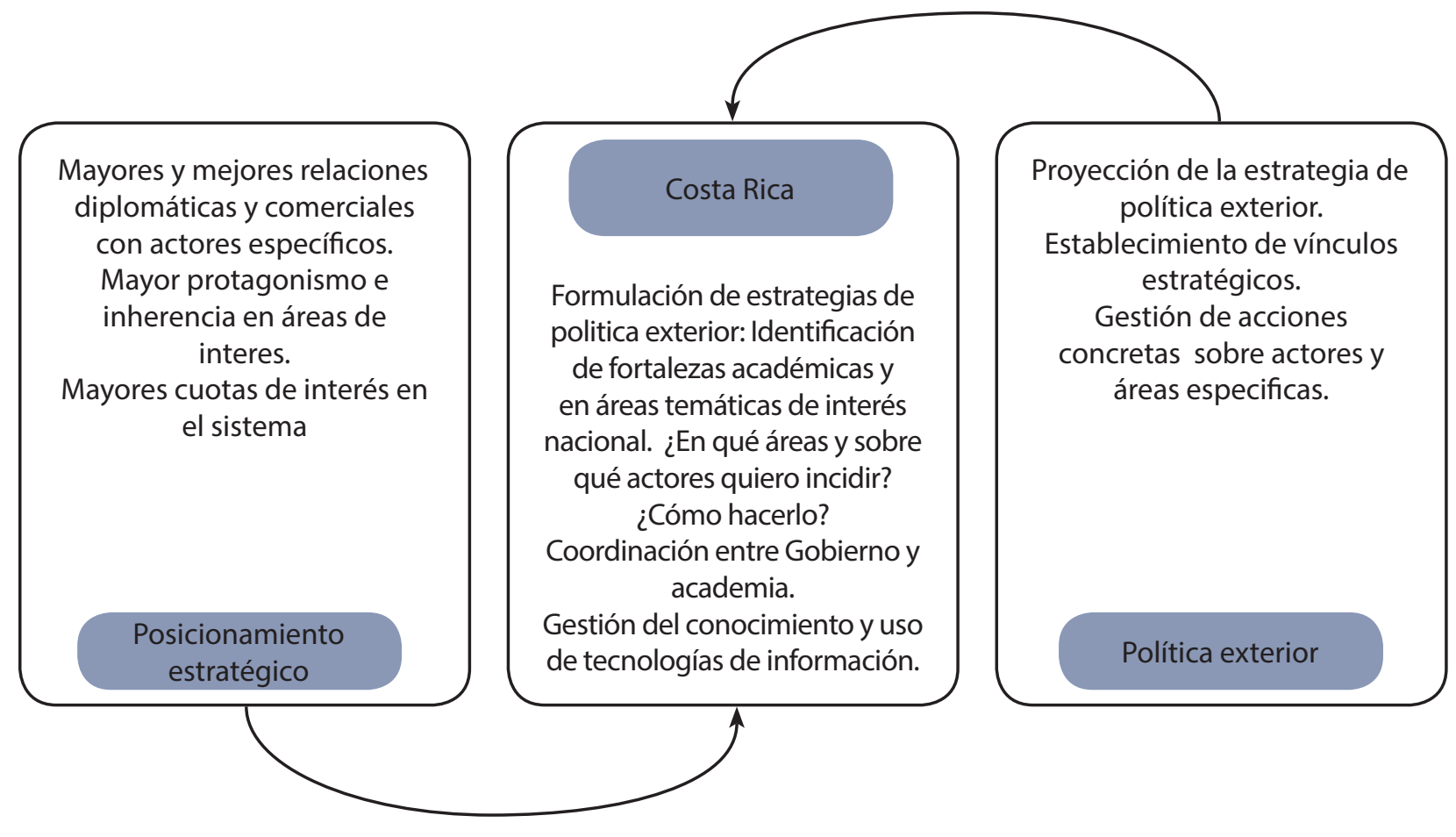

Figura 1. Teoría de sistemas: Costa Rica en Centroamérica. Elaboración propia.

Como puede verse, el conocimiento es el motor de gestión de muchas de las acciones del proceso en el marco del subsistema. En este punto, queda claro que la sinergia Universidad - Gobierno - Región es medular tanto para la claridad en términos de la gestión de la política exterior, como en lo referido al posicionamiento de los puntos estratégicos de agenda en el escenario internacional por parte de Costa Rica. En este sentido, la pregunta que se aborda en este trabajo es: Una propuesta para cambiar:

¿Qué factibilidad existe para el desarrollo de estrategias de política exterior mediante un trabajo coordinado y conjunto entre la academia costarricense de relaciones internacionales y el Ministerio de Relaciones Exteriores y Culto, donde el sector de educación superior tenga un rol protagónico? 


\section{Consideraciones metodológicas}

Desde la perspectiva sistémica, se ha buscado la comprensión de los agentes Estado y academia para la construcción de diferencias cualitativas en la generación de los outputs o estrategias de política exterior. De ahí que los esfuerzos metodológicos se hayan centrado, fundamentalmente, en lograr evidenciar la perspectiva de estos con respecto a los siguientes ejes, básicos desde la producción del alma del modelo sistémico, como lo es el conocimiento.

En el caso de la academia de Relaciones Internacionales costarricenses, se abordó, en primer lugar, el enfoque con el cual se autoproyecta en su rol de incidencia en el entorno nacional. Tal elemento es fundamental para determinar si dentro de su razón de ser, el nexo teórico existente entre el output y el agente está siendo asumido en prácticas concretas. Adicionalmente, se vieron los niveles de proyección de los conocimientos producidos, tratando de evidenciar si, potencialmente, este agente podría tejer estrategias de manera independiente. Es claro que en otros subsistemas esto ocurre con relativa facilidad y con mucha frecuencia. Además, se decidió establecer las posibilidades de vínculos funcionales o estratégicos en términos de producción o intercambio de conocimientos entre agentes de academias de relaciones internacionales en toda Centroamérica, partiendo de la posibilidad teórica de que tales agentes construyan comunidades aprendientes que logren, a partir de esta sinergia, incidencia concreta dentro del subsistema región centroamericana. Se quiso trabajar con el agente academia en la determinación de los niveles de relacionamiento de ésta con el agente cancillería costarricense $y$, fundamentalmente, se profundizó en la valoración que este agente tiene con respecto a su posicionamiento en la estructura país en materia de decisiones en el campo y en la gestión de conocimiento en la región.

En términos de fuentes, este agente fue representado a partir de los siguientes actores. Por un lado, personal coordinador de la carrera de Relaciones Internacionales de las Universidades Nacional de Costa Rica (UNA), Latina de Costa Rica (ULATINA), Internacional de las Américas (UIA) y Universidad Autónoma de Centroamérica (UACA), a los cuales se les aplicó una entrevista a profundidad durante los meses de mayo a junio del 2015. Además, se trabajó con personal académico de esta carrera de universidades públicas y privadas con una muestra no aleatoria de $n=10$ recuperada mediante la estrategia de muestreo de bola de nieve. Se les aplicó un instrumento cuantitativo de preguntas dicotómicas y categóricas de escala Likert y tourstone que abordaba los ejes descritos. Por otro lado, se realizó una indagación documental sobre el nivel de proyección de las universidades costarricenses, según el ranking web de universidades centroamericanas.

El segundo agente considerado en esta indagación fue la propia Cancillería que representa la acción organizada del Estado en términos de la construcción, difusión, posicionamiento y evaluación de la política exterior tanto en el sistema internacional, así como en el subsistema re- 
gión Centroamérica. Fueron cuatro los puntos considerados en la indagación realizada con el Canciller. El primero de ellos, relacionado con la identificación de los puntos o prioridades de la gestión de la política exterior costarricense en el marco de esta administración. En este sentido, el segundo elemento abordado trató de ubicar estos temas, tanto en la relación con el subsistema Centroamérica y otras regiones geográficas. De ahí, se tomó la decisión metodológica de abordar la perspectiva de la Cancillería con respecto al rol potencial que juega la academia de Relaciones Internacionales de Costa Rica en su relación con la Cancillería y, finalmente, la factibilidad de la construcción de una eventual estrategia de proyección del conocimiento producido en Costa Rica en este campo desde las Relaciones Internacionales para la generación de una estrategia de poder suave que permita impactar en términos de temáticas el subsistema Centroamérica. Estos ejes fueron abordados en una entrevista a profundidad con el señor canciller Manuel González.

El trabajo con las fuentes brindó información cuantitativa (instrumento aplicado a personal académico de la carrera de Relaciones Internacionales de universidades públicas y privadas) que fue procesada con Excel y SPSS. Además, la información cualitativa desprendida de las entrevistas fue procesada utilizando el software Dragon Naturally Speaking y el desarrollo de categorías de análisis mediante Nvivo.

\section{Consideraciones teóricas}

Gestión del conocimiento ha sido un término que ha tomado relevancia en la actualidad. Su conceptualización, según CEPAL,"responde a un proceso que se inicia con el tema de la Gestión por Competencias y el desarrollo de las TIC's para crear ventajas competitivas en economías que tienden a centrarse en el conocimiento y el aprendizaje" (Peluffo, B. \& Catalán, E. 2002, p. 14). Básicamente es:

(...) Una disciplina emergente que tiene como objetivo generar, compartir y utilizar el conocimiento tácito (Know-how) y explícito (formal) existente en un determinado espacio, para dar respuestas a las necesidades de los individuos y de las comunidades en su desarrollo. Esto se ha centrado en la necesidad de administrar el conocimiento organizacional y los aprendizajes organizacionales como mecanismos claves para el fortalecimiento de una región o espacio en relación con las visiones de futuro que van a determinar sus planes estratégicos de desarrollo en el mediano y largo plazo (Peluffo, B. \& Catalán, E., 2002, p. 14).

Eduardo Bueno (1999) asocia este término a un proceso más amplio como lo es la generación de capital intelectual en el marco de la sociedad del conocimiento. Desde su perspectiva, el identificar los múltiples niveles sistémicos que pueden ser impactados en virtud de producir conocimiento para la acción, incide en que empresas, organizaciones y actores sean entidades más competentes y que desarrollen capacidades para incrementar el valor de su propia organización, así como de los productos y servicios que ofrecen en la dinámica de mercado, mediante procesos de producción e innovación. Define la gestión del conocimiento en un pri- 
mer momento como dimensión operativa de la forma de crear y difundir el conocimiento entre miembros de una organización y también con demás agentes sociales en relación (1999).

Además explica el capital intelectual como la perspectiva estratégica de la empresa de reconocer sus propias fortalezas, competencias y procesos (intangibles) que incluyen al capital humano: capacidad de mejorar competencias entre personas y equipos; el capital estructural: el proceso de innovación; y el capital relacional: capacidad de mejora (Bueno, 2000). Es decir, el capital intelectual elige sus activos estratégicos, la gestión del conocimiento difunde el conocimiento a lo interno y externo de la organización, este se retroalimenta y genera nuevos insumos mediante el aprendizaje organizativo (AO), lo que genera amplias posibilidades de que la empresa pueda ser innovadora y competente en el mercado.

En este trabajo se comprende al agente comunidad académica de Relaciones Internacionales costarricense como una instancia de creación de capital intelectual que se encuentra en capacidad de generar estrategias de administración de su producto a fin de incrementar sus niveles de capital infra estructural y relacional en la interacción con otros agentes (en este caso Cancillería) en sistemas y subsistemas específicos (sistema internacional, subsistema Centroamérica). En otras palabras, el modelo de gestión del conocimiento (GC) es un proceso que no solo puede ser aplicado al nivel de empresas, sino que tanto universidades como Estados podrían ser comprendidos en función de él.

La sociedad se avecina hacia un momento cultural donde lo que rige al planeta es el pensar y el saber, como elementos conformadores de las estructuras de poder (Unesco, 2005). Esto no es nuevo, ya desde 1960 Peter F. Drucker indicaba que la edad post-industrial se caracterizaría por una estructura económica y social, en la que el conocimiento sustituiría materias primas, trabajo y capital como fuente de la productividad (como se citó en Kruger, 2006).

En esa misma línea, Bell, D. explicó la sociedad del conocimiento como la transición de una economía centrada en productos a una orientada a los servicios. En ella, el conocimiento teórico se convierte en la fuente principal de innovación y el punto de partida de los programas políticos y sociales. Tal movimiento genera la creación de una nueva tecnología intelectual como base de los procesos de decisión (como se citó en Kruger, 2006). Por estas razones, es que la GC y el Cl están altamente relacionados con el uso de las tecnologías de información en relación con la innovación y competitividad; por ello empresas, universidades y Estados no deben quedarse rezagados.

La forma de esta sociedad del conocimiento es la red. Según Unesco (2005), esta anatomía permite el desarrollo de una consciencia sobre las implicaciones de los problemas y soluciones globales de la totalidad de actores en el sistema internacional, ya que mediante la investigación científica y la educación, se puede compartir y mejorar la cooperación internacional y la colaboración científica y, con ello, encontrar nuevas soluciones a problemas globales. El auge de internet, la telefonía móvil y las tecnologías digitales -es decir, con la tercera revolución 
industrial- ha montado el soporte instrumental que permite la comprensión de la sociedad de la información y su énfasis en la generación de capital intelectual.

A la hora de visualizar el accionar de un Estado dentro de esta lógica, queda evidenciada la importancia estratégica de los procesos de innovación y competitividad, en una buena administración del conocimiento; debido a que, además de representar plataformas de crecimiento económico para el Estado, inciden directamente en posibilidad de acción de este; en el sistema internacional y en el subsistema regional. Ya en algunas regiones del planeta es posible visualizar fácticamente el modelo señalado. Según UNESCO, esto ha abierto nuevas oportunidades de desarrollo a los países del Sur:

La reflexión sobre las sociedades del conocimiento y su edificación permite replantearse el propio concepto de desarrollo. La nueva valorización del "capital humano" induce a pensar que los modelos de desarrollo tradicionales -basados en la idea de que eran necesarios inmensos sacrificios para alcanzar el crecimiento al cabo de largo tiempo y a costa de desigualdades muy considerables, e incluso de un profundo autoritarismo- están siendo substituidos por modelos basados en el conocimiento, la ayuda mutua y los servicios públicos. (2005, p. 20)

Es acá donde queda claro el rol potencial de las universidades en el proceso de construcción de una sociedad del conocimiento. Si intencionalmente estos actores toman medidas en las cuales, mediante una gestión y una administración adecuada de lo que producen se proyectan a la incidencia en procesos concretos para generar inputs de mayor valor agregado, tendrán potencialmente la oportunidad de participar en nuevas salidas que permitan la transformación o mantenimiento del sistema o subsistema. En otras palabras, tendrán la oportunidad de ganar protagonismo en la toma de decisiones nacionales e internacionales. Dado que el sistema internacional se avecina hacia una sociedad basada en el conocimiento, el hecho de que de las universidades hagan esto, genera un efecto dominó positivo, que permite que el país se proyecte internacionalmente como competente e innovador en ciertas áreas de conocimientos.

Con base en lo anterior, es posible enlazar la relación existente entre una adecuada gestión del conocimiento en una sociedad global que así lo exige, y la posibilidad de que los Estados nacionales tengan y gestionen mayores niveles de poder. En otras palabras, se parte, no de que el conocimiento es poder, sino de que este potencialmente empodera. Este no solo es una salida para el desarrollo, sino que se convierte en un insumo para tener mayor incidencia en el sistema internacional, sea a través de procesos de cooperación en materia de conocimiento, así como en la posibilidad de liderar estos procesos, al adquirir mayor prestigio internacional por poseer niveles importantes de producción científico-académica, o bien mediante estrategias de posicionamiento comercial o cualquier medio al alcance del Estado, que le permita al país tener mayor influencia internacional.

Por esa razón, es preciso que las academias costarricenses -en este caso de Relaciones Internacionales- logren una óptima gestión de su producción académica para que logren tener inherencia en 
la toma de decisiones nacionales. Asimismo, el gobierno debe estar consciente de la necesidad de incorporarlas en la toma de decisiones de política exterior, con el fin de profesionalizar su gestión, de manera que les permita adecuarse asertivamente al sistema internacional y a la sociedad del conocimiento. Es claro que, si en la sociedad del conocimiento el saber es el principal medio de la producción y la base de la futura estructura social y económica, entonces, también se traduce como poder.

En el sentido anterior, los países pueden llegar a generar los outputs específicos, para lograr objetivos concretos de acuerdo con sus intereses nacionales preestablecidos. En torno a esa lógica, una estrategia de posicionamiento internacional del conocimiento puede estar guiada por smart power ${ }^{1}$ (poder inteligente), de tal forma que se utilicen distintos medios de política exterior: diplomáticos, culturales, legales, comerciales, para alcanzar medios específicos. El académico Méndez (2012) expone en su trabajo "¿Poder inteligente?" como éste, desde una perspectiva académica, se visualiza como una combinación entre hard power y soft power y cuyo factor diferenciador es el uso de inteligencia contextual ${ }^{2} y$, además, requiere legitimidad internacional. Asimismo, desde una perspectiva política, es definido como una aplicación de poder sofisticada que conlleva una fusión entre influencia y poder, e implica como primer paso el uso de persuasión. Es una posibilidad de herramienta apropiada a cada situación, en la que se establece una relación entre medios (diplomáticos, culturales, legales, comerciales, militares etc.) y fines específicos. Es decir, "....se dispone de toda la gama de posibilidades para actuar en la escena internacional" (Méndez, M. 2012, p. 43).

Lo anterior ya ha quedado plasmado en la forma en que operan las universidades estadounidenses. Según un artículo titulado Soft power, hard power y sustainable smart power, se evidencia cómo, según el ranking London Times, las universidades ubicadas en los cinco primeros puestos son estadounidenses, además 75 de las primeras 200 también lo son (Dhanapala, J. 2011). Con ello han logrado atraer estudiantes de diversas universidades y países, para que puedan contribuir en la creación de conocimiento y proyectos. Esto ha manifestado la influencia que tienen universidades y Estados que ponen en práctica la gestión del conocimiento, para moldearlo internacionalmente. Es una forma de crear estrategias geopolíticas sobre la forma de pensar.

Desde esa lógica, surgen algunas preguntas: ¿Qué están haciendo los países y gobiernos nacionales para sacar provecho de las sociedades de conocimiento? ¿Están sacando partida y aprovechando procesos de gestión del conocimiento, apostando por una mayor cuota del poder en el SI? ¿Cómo una buena gestión del conocimiento puede generar mayores cuotas de poder en el sistema internacional?

$1 \quad$ Término de reciente data; desarrollado por Nye (2004) en su libro Soft Power. The Means to Success in World Politics.

2 Término mencionado por Nye (2008). Entendido como una “habilidad de diagnóstico intuitiva que ayuda/permite al líder alinear sus tácticas con sus objetivos para crear estrategias inteligentes en situaciones cambiantes", implica tanto la capacidad de discernir tendencias frente a la complejidad como adaptabilidad al tratar de influir en eventos o coyunturas. Conlleva: ... 1. Entendimiento del ambiente en constante evolución. 2. Capitalización de oportunidades (crear/generar suerte). 3. Ajustar el estilo (del líder) al contexto y necesidades de sus seguidores) (citado en Méndez, 2012, p. 34). 
Si bien no existe un manual general de cómo los países deben administrar su producción de conocimiento -pues cada uno se enfrenta a realidades distintas- es necesario que los gobiernos nacionales empiecen a tomar conciencia de la importancia de la gestión de conocimiento como pilar de desarrollo y crecimiento nacional. Ante ello; el aspecto coyuntural y las características estructurales de cada Estado, juegan un papel trascendental a la hora de que se quiera trazar una hoja de ruta para el desarrollo de procesos de gestión del conocimiento.

Montobbio, M. (2013) establece relaciones entre conocimiento y poder en el sistema internacional, en su libro Geopolítica del pensamiento. En él ha sabido ejemplificar el poder internacional que han adquirido países como: España, Estados Unidos, Singapur, Brasil, Unión Europea mediante la proyección de conocimiento sobre áreas en las que son fuertes. Una de las apuestas frecuentes en España, por ejemplo, es la creación de think tanks ya que estos "pueden contribuir decisivamente a la creación del espacio público" (2013, p. 18), por su autopercepción como fábricas o laboratorios de ideas, dedicados a influir y aportar inputs a la elaboración de políticas públicas. Esta lógica se plantea en la disyuntiva de que en el sistema internacional: 0 te piensas o te piensan (Montobbio, M. 2013). Por lo que es mejor incidir en el sistema; a que el sistema y sus actores más poderosos incidan sobre un determinado país.

Países como los centroamericanos, y en particular Costa Rica, que no poseen gran variedad de centros generadores de think tanks, pueden empezar a sacar provecho de sus universidades - las cuales existen en abundancia- en temáticas políticas, económicas, de integración regionalo bien de cualquier tema exterior sobre el cual el país quiera empezar a incidir; empero, es preferible un tema emergente en donde no se haya profundizado mucho en la región y Costa Rica pueda ofrecer un valor agregado- ya que el conocimiento adquiere la condición de producto o servicio en un sistema que es regido por el conocimiento.

Haciendo alusión a la idea ya planteada, Montobbio deja en entredicho como la proyección de conocimiento se puede convertir en una herramienta de poder: "... el incremento de nuestro poder de pensamiento constituye, en definitiva, uno de los factores de actoría internacional que mejor puede sustituir la disminución de otros y por ello contribuir mejor al mantenimiento o incremento de nuestro poder internacional global" (2013, p. 12). Es claro, a partir de estas consideraciones, que:

1. La sociedad: a) está en un momento clave en que se avecina hacia una sociedad regida por el conocimiento. b) Que la gestión y buena administración del conocimiento aplicado a la política exterior de un país es una herramienta clave para el incremento de poder en el sistema internacional. c) Que para ganar mayor poder internacional a través del conocimiento, es necesario crear una estrategia de smart power, según los intereses del país. d) Que esa estrategia se debe traducir en outputs específicos, que al interactuar con el sistema le generen los inputs deseados al país. 
2. Aunado a lo anterior, es claro que para lograr una estrategia bien articulada de diseño de política exterior, donde se involucre al sector académico, la investigación científica y capital humano-intelectual-estructural de alta calidad, se deben hacer esfuerzos gubernamentales importantes, para trabajar con ambos sectores. Dicho esfuerzo puede conllevar los siguientes beneficios:

a) La posibilidad de crear gobernanza ${ }^{3}$ sobre ciertos procesos internacionales sobre los que se tiene conocimiento y experiencia -en el entendido de que quién tiene la información tiene poder. b) Crear vínculos estratégicos en materia de cooperación internacional: en el aspecto científico-académico, permitiendo la posibilidad de legitimarse como un país referente en la materia. c) Jugar con estrategias de poder inteligente a través de la diplomacia (diplomacia pública, ciudadana, vínculos culturales, legales, etc.) y de una estrategia clara de política exterior, que pueda conllevar no solo beneficios políticos, sino comerciales.

\section{Presentación de resultados}

\section{Hallazgos en el agente academia de Relaciones Internacionales de Costa Rica}

A continuación se presentarán los resultados más relevantes en torno a la investigación realizada en universidades. Estos son los insumos recogidos a partir de la entrevista: Poder inteligente, conocimiento y academia, realizada a coordinadores y coordinadoras de carrera:

Grooscor, G. (2015). Entrevista coordinación UACA: Poder inteligente, conocimiento y academia. Entrevista realizada 20 de mayo, San José, Costa Rica.

Rodríguez, P. (2015). Entrevista coordinación ULATINA: Poder inteligente, conocimiento y academia. Entrevista realizada del 05 de mayo, San José, Costa Rica.

Suárez, M. (2015). Entrevista coordinación UIA: Poder inteligente, conocimiento y academia. Entrevista realizada el 14 de mayo, San José, Costa Rica.

Flores, F. (2015). Entrevista coordinación UNA: Poder inteligente, conocimiento y academia. Entrevista realizada el 13 de mayo, Heredia, Costa Rica.

Asimismo, se aplicó la misma entrevista 10 sujetos del personal académico de la Universidad Nacional de Costa Rica. El instrumento utilizado fue el mismo para todas las personas entrevistadas, por lo que en esta sección se exponen, en términos porcentuales, las posiciones de la "academia" de Relaciones Internacionales como colectivo (y no de manera individual) sobre temas puntuales.

3 Definido como la capacidad que tienen ciertos actores para guiar y sancionar los comportamientos de un colectivo (veáse Donahue, J \& Nye, J. [2000] en Governance in a globalizing world).

Luis Diego Salas Ocampo y Fabiana María Jenkins Arias, 
El primer elemento del que se tiene un impacto clave, en lo referente a la calidad del producto generado por este agente, está asociado con la autopercepción del nivel de incidencia de las decisiones en política exterior. En este sentido, tanto personal coordinador de carrera en su conjunto, así como la totalidad del personal académico abordado, estuvieron de acuerdo en señalar que la incidencia es completamente baja.

Este dato es coincidente con otras investigaciones en el campo, generadas en el proyecto FOCAIS. Alpízar, M. \& Salas, L. (2015) han señalado la ausencia de elementos comunes en el enfoque con el que se asumen las Relaciones Internacionales en Costa Rica, particularmente continúan en las temáticas donde se va desde la perspectiva técnica en comercio internacional, hasta la dimensión más formalista en el campo del derecho.

Este hallazgo permite dejar claro que, desde la perspectiva del personal participante abordado, la incidencia de la disciplina de las Relaciones Internacionales es baja en la toma de decisiones gubernamentales en materia de política exterior. Esto tiene dos posibilidades prácticas: la primera relacionada con el eventual autismo institucional que podría tener la Cancillería con respecto a los insumos que la academia produzca; o bien, la imposibilidad o parálisis temática que podría tener el agente academia para la reflexión y acción sobre temas estratégicos. Será importante, en estudios posteriores, la incorporación también de la coordinación del Instituto Manuel María Peralta, a fin de determinar si esta potencialmente está cumpliendo esta labor de generación de conocimientos para la incidencia.

Es claro que esta distancia impacta el diseño, análisis y ejecución de outputs en la materia de política exterior. ¿Qué está impidiendo que haya una labor de coordinación entre ambas esferas? ¿Por qué el gobierno no aprovecha el capital intelectual y humano que producen sus universidades?

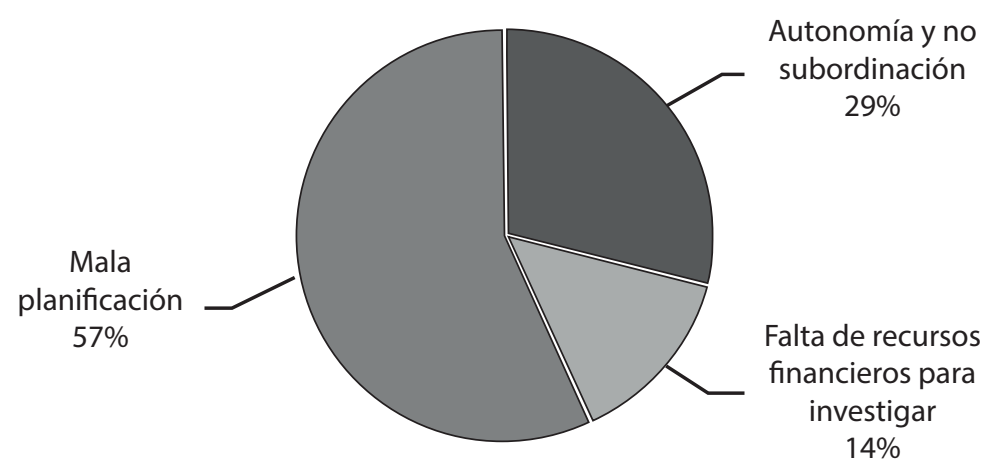

Figura 2. Causas del distanciamiento entre el gobierno y la academia, según informantes del agente academia.

Fuente: elaboración propia según entrevista a personal coordinador y académico de relaciones internacionales de universidades costarricenses durante el primer semestre del 2015. 
Son tres las razones identificadas para explicar la distancia. En primer lugar, se indica la "deficiente planificación" y, en segundo lugar, elementos relacionados con la autonomía de las instancias y el no querer estar bajo niveles de subordinación con respecto al gobierno. Como última razón se indican problemas de presupuesto para la investigación.

Resulta evidente que, pese a que la actual administración ha tenido en la conformación de equipo de gobierno una importante ala de la academia (particularmente de la Universidad de Costa Rica y la Escuela de Economía de la Universidad Nacional), no hay una estrategia marcada de gestión del conocimiento producido por este ente para el desarrollo de política exterior. De ahí que la planificación deficiente sea, fundamentalmente, por la ausencia dentro de los procesos de planificación, o por una decisión de acercamiento. Llama la atención, sin embargo, el hecho de que, en los últimos meses, la Cancillería ha firmado un convenio de cooperación y acercamiento con la Universidad Nacional.

Lo interesante del caso es que aparezcan, en este nivel, los temas de autonomía y de ausencia de fondos para la investigación. Es posible que estos dos elementos evidencien la presencia de feudos de comodidad institucional. Por un lado, el agente academia podría considerar mucho más seguro seguir navegando en sus mismos nichos temáticos, que entrar en la arena de las decisiones de política exterior, potencialmente por miedo a reconocer que debe refrescar enfoques, teorías y metodologías. Además, podría existir el temor, desde la Cancillería, de que elementos de la cultura académica costarricense (particularmente los manejos de los tiempos de producción de conocimiento) impregnen los procesos de acción institucional.

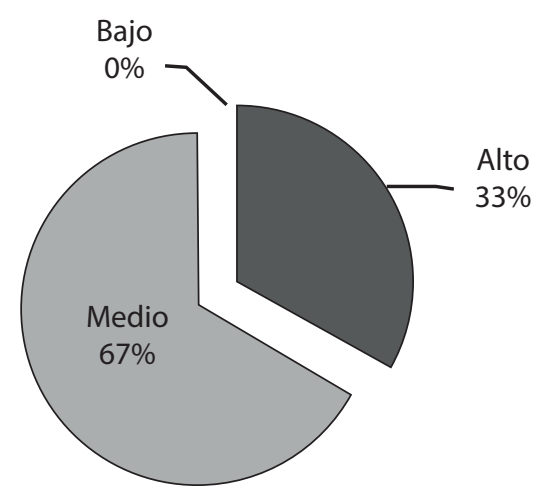

Figura 3. Nivel de calidad de los productos académicos e investigativos de internacionalistas costarricenses en relación con el resto de Centroamérica. Fuente: elaboración propia según entrevista a personal coordinador y académicos de relaciones internacionales de universidades costarricenses. 
La figura 2 evidencia que el temor institucionalizado nace posiblemente de la diferencia de las lógicas de trabajo. Los propios actores del agente academia reconocen que el nivel de calidad de lo que producen es medio. La pregunta es: ¿Qué causa que su propio trabajo sea percibido de esta forma? Se ha encontrado que la implantación de modelos teóricos y metodológicos de la disciplina, sin los procesos de "tropicalización" puede generar una ausencia de respuestas a los temas más puntuales (Alpízar, M. \& Salas, L., 2015), en particular y como demuestra el gráfico, al subsistema más concreto de acción de la política exterior costarricense, como lo es el subsistema Centroamérica. Lo interesante es que al indagar sobre el nivel de posicionamiento de la producción académica sobre Centroamérica en las Relaciones Internacionales, los datos obtenidos fueron los que se exponen en la figura 4.

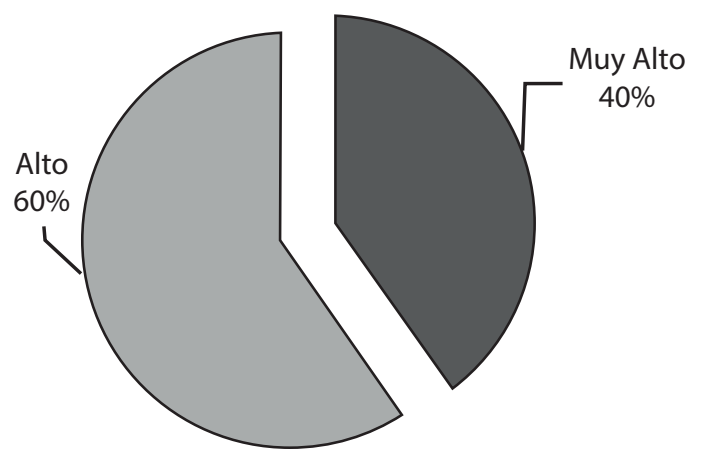

Figura 4. Nivel de importancia del estudio de la región centroamericana en las Relaciones Internacionales costarricenses. Fuente: elaboración propia según entrevista a personal coordinador y académico de Relaciones Internacionales de universidades costarricenses, realizada durante el primer semestre de 2015.

Los actores del agente academia, de manera interesante, indican la existencia de un alto nivel de importancia en su análisis de la región centroamericana. Esto lleva inmediatamente a las preguntas: ¿Sobre qué investigan? ¿Para qué lo hacen? Esclarecer estas dudas permite imaginar estrategias para proyectar el conocimiento generado hacia quienes tienen a cargo la toma de decisiones de la región, lo que potencialmente traería posibles beneficios para el país en materia de posicionamiento y para Centroamérica en materia de cooperación, intercambios, y crecimiento investigativo y académico. 


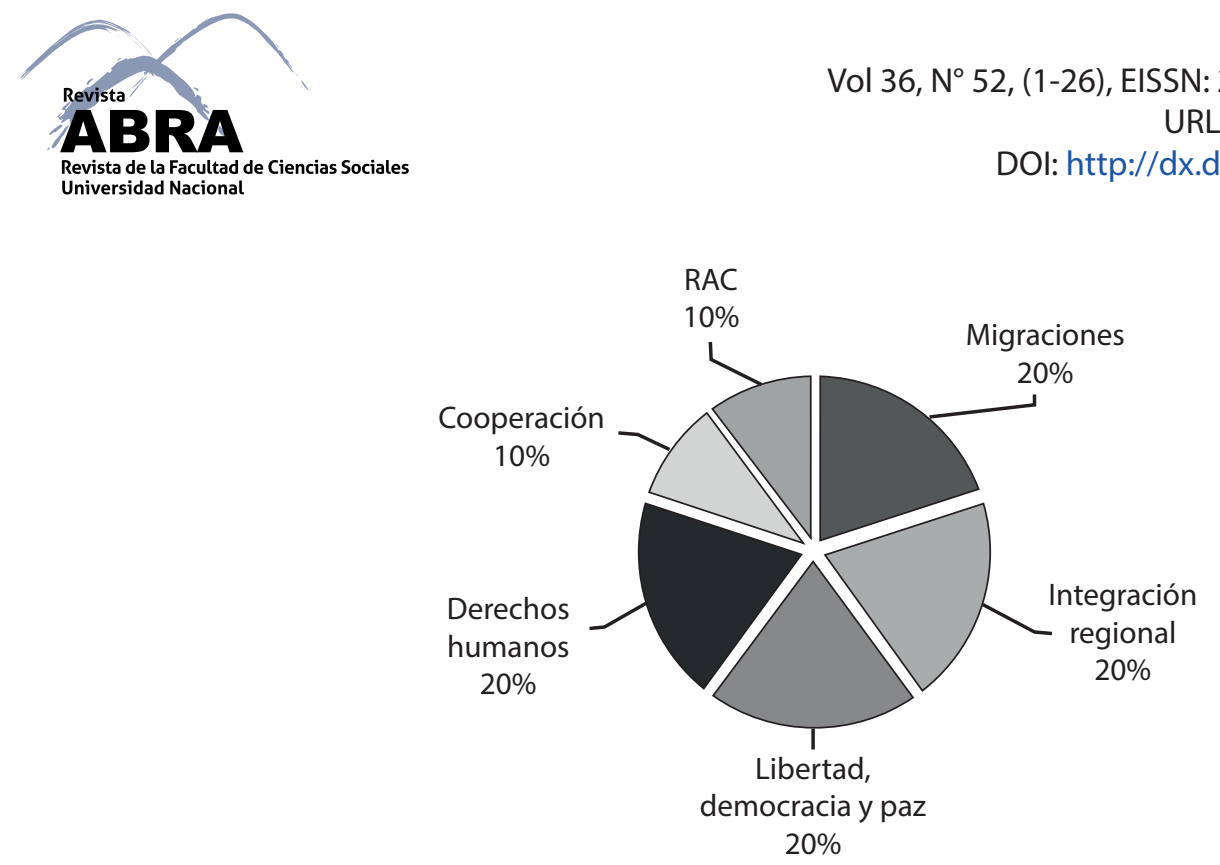

Figura 5. Temáticas de estudio en que ha destacado la disciplina costarricense de las Relaciones Internacionales en Centroamérica. Fuente: elaboración propia según entrevista a personal coordinador y académico de relaciones internacionales de universidades costarricenses, realizada durante el primer semestre de 2015.

Según el personal académico y coordinador entrevistado, los temas en que históricamente Costa Rica ha destacado son: Derechos humanos; libertad, democracia y paz; integración regional y migraciones. Cada categoría representó un $20 \%$ de personas entrevistadas. Por su parte, la cooperación internacional y la Resolución alternativa de conflictos (RAC) tuvieron un 10\%, respectivamente. Llama la atención cómo prácticamente 4 de los 6 temas macros señalados tienen una fuerte cercanía con la dimensión jurídica y formal del ejercicio de las RI y que temas, quizá de naturaleza más operativa y práctica, constituyan solamente un tercero del total de intereses señalados. Una pregunta que queda en el aire y para la cual la evidencia no permite respuesta es: ¿Son las Relaciones Internacionales una disciplina en extremo formalista que no permite en su estado actual al agente academia acercarse a la generación de insumos para la toma de decisiones del país?

Sería posible pensar que, en la medida en que existiesen vínculos investigativos y operacionales con otras universidades de la región, esta tendencia podría, o bien, reajustarse; o bien, ser retroalimentada por la tradición de trabajo de otros países. No obstante, prácticamente la mitad de sujetos entrevistados indicó que las carreras de relaciones internacionales no poseen vínculos funcionales o investigativos con otras casas de estudio de la región que impartan la disciplina. Esta misma proporción indica la presencia de vínculos entre las carreras de Relaciones Internacionales de Costa Rica con la Cancillería, tal y como evidencia la figura 6. 


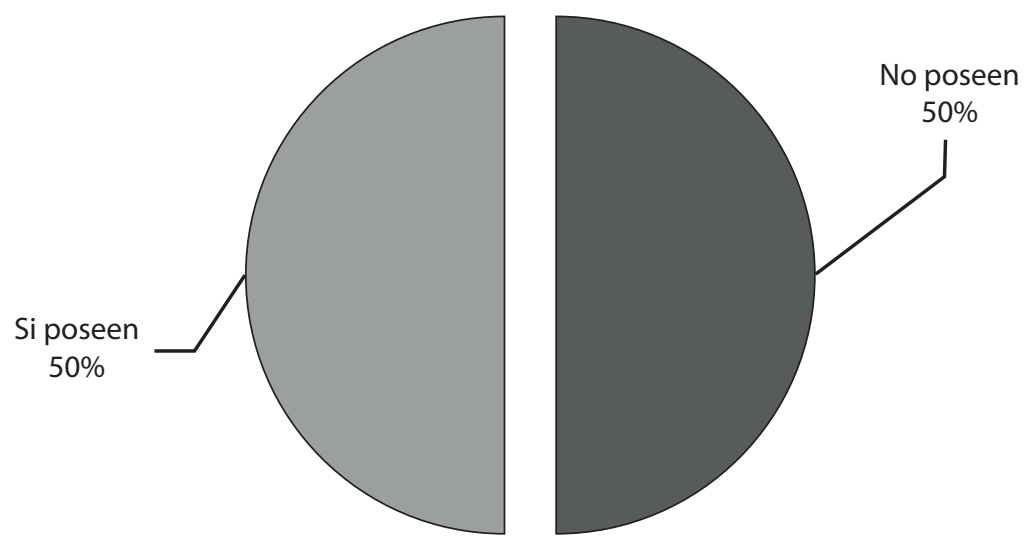

Figura 6. Porcentaje de universidades costarricenses que imparten R.R.I.I con vínculos académicos con universidades centroamericanas. Fuente: elaboración propia según entrevista a personal coordinador y académico de Relaciones Internacionales de universidades costarricenses, realizada durante el primer semestre de 2015.

Resulta evidente el hecho de que, potencialmente, es posible imaginar que el centro de la existencia de las carreras de Relaciones Internacionales es graduar profesionales y no necesariamente alimentar procesos de proyección para la incidencia en las estrategias de política exterior. Esto es sugerente en la medida en que, históricamente, uno de los principales empleadores de este tipo de profesionales ha sido el propio Estado. Ahora bien, queda también planteada la duda sobre la direccionalidad que tendrán estos profesionales, sobre todo en el marco de que los niveles de formación suelen tener una alta concentración teórica. El gráfico 6 da cuenta de áreas de interés potencial que desde el agente academia se visualizan como estratégicos para difundir su conocimiento en el subsistema región.

Además, es interesante que los dos valores principales que perciben el personal académico y coordinador de carrera apuntan a que la academia debe seguir haciendo lo mismo en la región, salvo una importante e interesante diferencia que tiene que ver con el tema de medio ambiente. El dato es sugerente, dado que es innovador, responde a necesidades nacionales y regionales muy específicas y, además, en el que Costa Rica ha destacado en la región y en el mundo en el campo de producción de conocimientos. Ejemplo de ello es cómo Costa Rica es país oferente de cooperación técnica en medio ambiente. Además, "Costa Rica ha logrado desarrollar experiencias y conocimientos en diversas áreas del desarrollo, entre los que destacan biodiversidad, ecoturismo, salud, educación, entre otras, convirtiéndose en un país de referencia en América 
Latina y el mundo" (MIDEPLAN, 2010, p.11). Asimismo, ha sido un área utilizada desde la gestión de la política exterior del subsistema región, sobre todo en el marco del conflicto más reciente de este país, como lo fue el caso con Nicaragua, sobre Isla Calero.

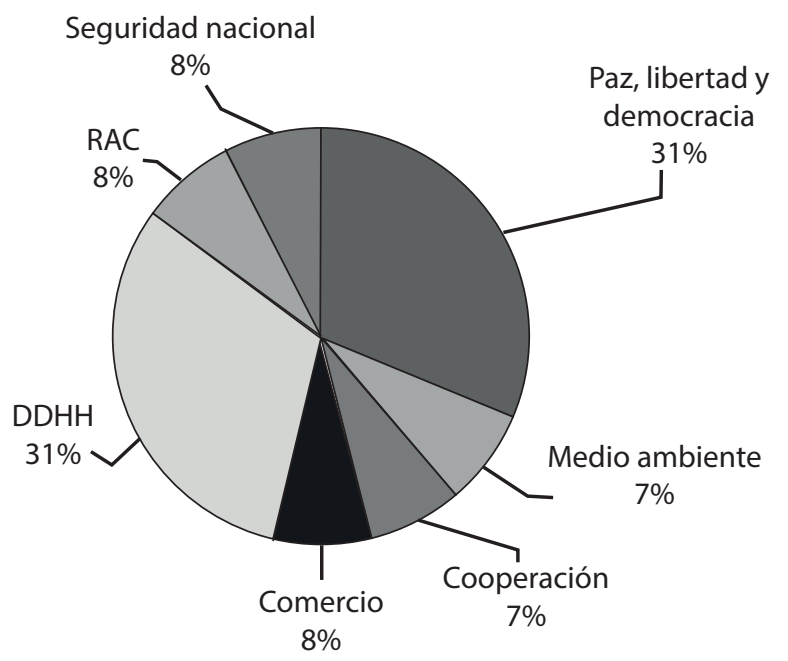

Figura 7. Temas estratégicos en que el país debe enfocar la gestión y difusión del conocimiento en Centroamérica. Elaboración propia según entrevista a personal coordinador y académico de Relaciones Internacionales de universidades costarricenses, realizada durante el primer semestre de 2015.

Este tema podría ser considerado una ventana de oportunidad para orientar un esfuerzo organizado de cara a la incorporación del agente academia en la producción de imputs de conocimiento que den como consecuencia la generación de outputs en el subsistema Centroamérica para fortalecer la incidencia de la academia. No obstante, debe indicarse que ninguna de las entidades de oferta de la carrera de relaciones internacionales en Costa Rica consultadas poseen estrategias para posicionar sus productos académicos e investigativos en la región, tampoco aplican estrategias de gestión de conocimiento. El 100\% de las unidades reconoció no poseer estrategia de internacionalización de los conocimientos producidos desde la disciplina de las relaciones internacionales. No obstante, también la totalidad de informantes consideró que esto es medular para el país y para el agente academia y que, por lo tanto, debería trabajarse en esa línea. 


\section{Hallazgos del agente gobierno}

A continuación se presentarán los resultados más relevantes en torno a la posición gobierno sobre los ejes de indagación. Esto, en la figura y en el criterio del jerarca superior de la Cancillería de la República, don Manuel González Sáenz, a quien se entrevistó con respecto a la temática: Poder inteligente, conocimiento y política exterior (Comunicación personal del 13 de mayo de 2015, en San José, Costa Rica).

Un primer elemento que se desprende del análisis de la información recopilada con este informante es la cercanía existente entre los temas definidos como especialmente importantes por la academia y la tradición histórica de trabajo de este ente en los temas regionales. Según este, Costa Rica ha destacado en Derechos humanos, respeto al derecho internacional y en paz, democracia y desarme. Esta cercanía identificada coloca nuevamente en la agenda el tema de la formación de profesionales y los requerimientos concretos que tiene esta instancia para que los conocimientos producidos por la academia sean utilizados para la toma de decisiones. Con respecto a los temas en los que debería insertarse el país, tampoco hay grandes sorpresas en lo que es la visualización de una voluntad de seguir en la misma línea, salvo también la incorporación del tema ambiental (M. González, comunicación personal, 2015), tal y como destaca en la figura 8.

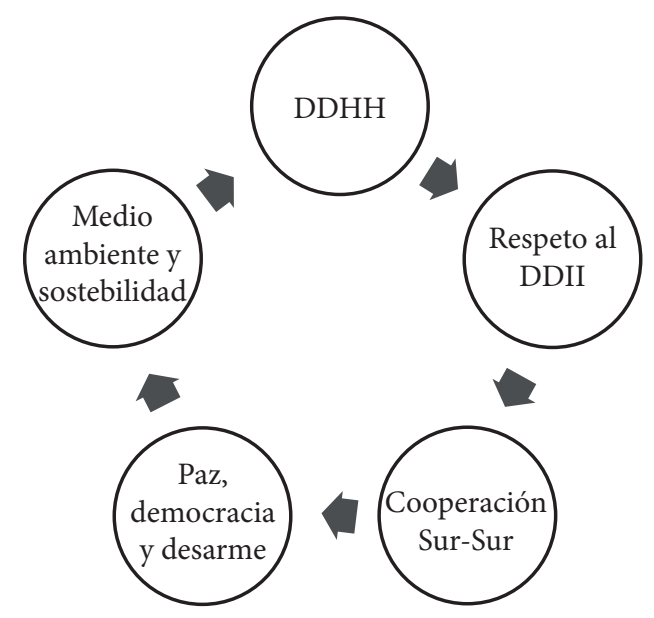

Figura 8. Áreas prioritarias en que el país debe destacar en Centroamérica a través de su política exterior. Elaboración propia basada en entrevista realizada al Canciller de la República de Costa Rica, Manuel González Sáenz, 13 de mayo de 2015. 
Los temas destacados por el Canciller en la entrevista encajan con perfecta exactitud respecto a los pilares de la política exterior, definidos por esta administración. El quinto pilar es: "la promoción del desarrollo sostenible, en sus tres aproximaciones la social, económica y el ambiental- y la coordinación y representación política en las negociaciones ambientales internacionales" (M. González, 2014, p.1). Además destacó en ese mismo documento "Desafíos de la política exterior costarricense: Hacia la consolidación de una política de Estado", que dentro de los círculos concéntricos donde Costa Rica debe tener influencia en América Latina, Centroamérica -como colectivo- se ubica en el tercer lugar, de 7 lugares (M. González, 2014).

Por otra parte, durante la entrevista con el Canciller destacó de manera importante la actitud propositiva que como jerarca tiene en relación con estas consideraciones que la teoría permite en términos de la gestión de conocimiento. Sin embargo, las ideas planteadas, potencialmente, distan mucho de los mecanismos de relacionamiento o de enfoque de acción que la academia ha tenido. En la siguiente tabla 1 se resumen las líneas que el jerarca proyecta a partir de la relación con la academia.

Tabla 1

Beneficios país: Acercamiento entre la Cancillería y la disciplina de relaciones internacionales

1. Posibilidad de crear un departamento de inteligencia que diseñe estrategias propositivas sobre las posiciones que debería de tomar el país ante dinámicas internacionales específicas.

2. Posibilidad de crear estrategias de acercamiento bilateral con socios estratégicos. ¿Cómo debe ser el acercamiento? ¿En qué etapas? ¿En qué áreas?

3. Posibilidad de elaborar indicadores para medir la eficacia y eficiencia de la política exterior costarricense, al evaluar el trabajo de la Cancillería, embajadas, consulados y el contacto directo con individuos.

Fuente: elaboración propia basada en entrevista realizada al canciller de la República Manuel González, 2015.

Resulta claro que las tres ideas que este esboza (departamento de inteligencia, estrategia de acercamiento de socios y gestión de indicadores) se mueven en el plano instrumental. Es posible identificar, entonces, una línea de preocupación por la generación de conocimiento aplicado. Dicho en otras palabras, pese a que desde el punto de vista teórico, el agente academia ha desarrollado mucho conocimiento para aumentar la comprensión de los fenómenos, la preocupación, en este caso de gobierno, tiene que ver con la generación de herramientas para solucionar problemas o para promover procesos. De ahí se desprende una duda fundamental relacionada con ¿cuál es la tradición país en la conformación de equipos intelectuales que construyan conocimiento para la toma de decisiones y para la dotación de instrumentos? 


\section{Hallazgos sobre las posibilidades del agente academia para la construcción de equipos intelectuales}

Como complemento a los hallazgos anteriores, se consideró de vital importancia investigar sobre el prestigio internacional y regional de las universidades costarricenses -incluidas las involucradas en este estudio- así como de otros centros generadores de conocimiento, que juegan el papel de think tanks. En torno a ello, se ha logrado constatar que las universidades públicas costarricenses gozan de prestigio a nivel internacional, principalmente a nivel de Centroamérica y América Latina. Para el año 2014, un ranking de 1295 universidades (Ranking Web de Universidades, 2014), posicionó a las costarricenses en los siguientes puestos (tabla 2).

Tabla 2

Universidades públicas en Centroamérica

\begin{tabular}{ll}
\hline UNIVERSIDAD PUESTO & Posición \\
\hline Universidad de Costa Rica (UCR) & 2 \\
\hline Universidad Nacional de Costa Rica (UNA) & 12 \\
\hline Universidad Estatal a Distancia (UNED) & 70 \\
\hline Instituto tecnológico de Costa Rica (TEC) & 95 \\
\hline Universidad Técnica Nacional (UTN) & 248 \\
\hline TOTAL DE UNIVERSIDADES & 1295 \\
\hline
\end{tabular}

Fuente: elaboración propia según Ranking web de universidades sobre América Central y el Caribe (2014).

Si se pondera la ubicación de las universidades públicas costarricenses con base en los datos de este ranking, se estaría en presencia de que estas ocuparían aproximadamente el lugar 85 . Esto evidentemente se ve influenciado por dos puntos extremos, el primero de ellos, el hecho de que la Universidad de Costa Rica ocupa el segundo lugar de todo el ranking centroamericano y, por supuesto, el hecho de que la Universidad Técnica Nacional de reciente data, no tiene aún un conjunto de elementos estratégicos, lo cual incide en su calificación.

Ahora bien, si este ejercicio se hace solamente con las universidades que ofrecen la carrera de Relaciones Internacionales se tiene, por un lado, que para el caso costarricense prácticamente todas salvo la UNA, son de corte privado. Esto tiene un impacto en el ranking centroamericano, tal y como se visualiza en la tabla 3. 
Tabla 3

Universidades que imparten RRII en Costa Rica

\begin{tabular}{lr}
\hline UNIVERSIDAD & PUESTO \\
\hline Universidad Nacional de Costa Rica (UNA) & 12 \\
\hline Universidad Latinoamericana de las Ciencias y el Arte (ULACIT) & 115 \\
\hline Universidad Latina de Costa Rica & 146 \\
\hline Universidad Autónoma de Centroamérica (UACA) & 188 \\
\hline Universidad Internacional de las Américas & 391 \\
\hline TOTAL DE UNIVERSIDADES & 1295 \\
\hline
\end{tabular}

Fuente: elaboración propia según Ranking web de universidades sobre América Central y el Caribe (2014).

Si se realiza el cálculo antes señalado, solamente para las universidades que ofrecen la carrera de Relaciones Internacionales en Costa Rica, se cae del lugar 85 al 170. En otras palabras, es claro que existe un impacto de calidad desde esta perspectiva, el cual debe ser valorado y pesado para cualquier estrategia donde se quiera incorporar al agente academia con gobierno para la definición de outputs en términos de política exterior. Esto también tiene un impacto sumamente importante en la generación de inputs, ya que tal y como se conoce actualmente en el escenario nacional, las instituciones de educación privada no cuentan con ningún tipo de recurso del Fondo de Educación Superior (FEES) para la generación de investigación y no necesariamente han visto la ventana de oportunidad de este tipo de iniciativa como área rentable de negocio.

Es evidente que en la actualidad no es en la educación privada donde existen las condiciones para la generación de equipos intelectuales de venta de ideas y gestión de conocimientos, ya que existen limitantes estructurales para el funcionamiento de este mismo, en vista de que estas instituciones de educación no necesariamente construyen procesos sostenidos en el tiempo con su personal académico, que no vayan más allá de impartir sesiones; pero, sin duda, esta sería una ventana de oportunidad.

En un estudio internacional ya un tanto antiguo, pero el único identificado en este marco, se valoró la posición de think tanks mundiales, latinoamericanos y centroamericano. En el caso costarricense, se mostró como único centro con estas características a la Facultad Latinoamericana de Ciencias Sociales Costa Rica. Este tuvo calificaciones sumamente destacas en los temas de ideas generales sobre ciencias sociales; innovación; en relaciones exteriores y en participación pública; etc.

En este punto y en relación con los hallazgos mostrados con anterioridad, resulta interesante observar como FLACSO ni ningún otro think thank costarricense aparece en la categoría medio ambiente. Por su parte, para América Latina solo destacan en medio ambiente, el Centro 
Brasileiro de Relaçoes Internacionais en Brasil; y el Centro Mexicano de Derecho Ambiental en México. Es decir, en la región centroamericana no existe ningún think tank que destaque en esta categoría; por lo tanto, el medio ambiente se presenta como un espacio innovador y emergente en el que las universidades costarricenses así como las academias de Relaciones Internacionales y el gobierno puede sacar partida para proyectarse internacionalmente.

Los insumos presentados en este apartado permiten determinar que en el campo de las relaciones internacionales, en la creación de equipos de investigación con potencial de jugar un papel potencial, como Think Thank en el caso costarricense, son escasos.

Tabla 4

Posiciones internacionales de FLACSO Costa Rica

\begin{tabular}{cccccc}
\hline ÁREA & General & $\begin{array}{c}\text { En ideas } \\
\text { innovadoras }\end{array}$ & $\begin{array}{c}\text { Relaciones } \\
\text { exteriores y } \\
\text { participación } \\
\text { pública }\end{array}$ & $\begin{array}{c}\text { Afiliados a } \\
\text { universidades }\end{array}$ & $\begin{array}{c}\text { Impacto a } \\
\text { políticas } \\
\text { públicas }\end{array}$ \\
\hline CENTROAMÉRICA & 1 & 1 & 1 & 1 & 1 \\
LATINOAMÉRICA & 4 & 5 & 6 & 2 & 8 \\
GLOBAL & 74 & 51 & 44 & 29 & 63 \\
\hline
\end{tabular}

Fuente: elaboración propia basada en datos tomados del artículo “Geopolítica del conocimiento" (Montobbio 2013).

Es posible la existencia de un problema de administración en lo concerniente a los mecanismos de gestión de conocimiento en las universidades para la incidencia en la generación de outputs a nivel país. Resulta medular para el acercamiento en esta dirección, la discusión sobre el qué enseñar, para qué hacerlo y cómo capitalizarlo en los problemas estratégicos país en materia de política exterior.

\section{Discusión}

Plantear una estrategia para el posicionamiento del conocimiento costarricense en la región desde las Relaciones internacionales, presenta un conjunto de retos desde el punto de vista académico y político realmente interesantes:

Es claro que la creación de esa estrategia, gestionada desde el gobierno en coordinación con la academia, debe desarrollarse con el objetivo no solo de darle mayor protagonismo al 
sector académico costarricense en el diseño y gestión de políticas públicas, sino también de generar outputs o salidas estratégicas de conocimiento, que una vez, al circular en el sistema centroamericano, generen retroalimentación positiva y, con ello, inputs o entradas beneficiosas al país. Se trata de usar el conocimiento desde una visión geopolítica: ¿Dónde puede ser posicionado? ¿Qué deficiencias se captan en el sistema o subsistema? ¿En cuáles de esas áreas puede aportar el país?" ¿A la luz de cuáles serían los beneficios a largo plazo? ¿Desde dónde debe comenzarse?

Se piensa que el tema medular tiene que ver con cómo ganar poder como país; una estrategia de internacionalización del conocimiento desde el poder inteligente consiste en generar una buena reputación y proyección internacional, lo cual, desde el punto de vista comercial, es clara y altamente comercializable: se forja la base para posicionar el conocimiento como un servicio necesario, que Costa Rica tiene el potencial de producir, posicionar y vender.

Ante ello, en los últimos años, el sector de política comercial exterior ha mostrado una gran capacidad adaptativa y, sobre todo, una gran posibilidad de construir acuerdos colectivos con los diferentes sectores para el posicionamiento del país en ciertos mercados. El punto de unión con el poder inteligente y con la cancillería tendría que ser, necesariamente, el tema ambiental, sobre el cual existen acuerdos tanto a nivel de Ministerio de Relaciones Exteriores y Culto, Ministerio de Comercio Exterior, Ministerio de Ambiente y Energía y Ministerio de Planificación y Política Económica. Solo por mencionar dos ejemplos, la marca País y el Catálogo de oferta de la cooperación técnica de Costa Rica, son producto de ello. Por último, el canciller Manuel González Sáenz mencionó, como uno de los pilares de política exterior de la actual administración: «La promoción del desarrollo sostenible, en sus tres aproximaciones -la social, económica y ambiental- y la coordinación y representación política en las negociaciones ambientales internacionales» (M. González, 2014).

Resulta relevante, en el sentido anterior y según el Vigésimo Informe del Estado de la Nación (20142015) sobre política exterior, cómo en la Administración Solís Rivera se han manifestado iniciativas importantes para unificar los esfuerzos de la Cancillería y el Ministerio de Relaciones Exteriores, en cuanto a la definición de la línea de política exterior; sobre todo, en torno al tema de la adhesión de Costa Rica a la OCDE y a la Alianza del Pacifico. No obstante, se señala como aún existen vacíos en la definición de la rectoría o línea jerárquica de los procesos (Cascante, 2014, citado en Vigésimo Informe del Estado de la Nación). Es allí donde queda mucho por realizar a la hora de coordinar el trabajo de dichas instituciones y el tema de medio ambiente y sostenibilidad parece ser uno de ellos.

Si el tema de ambiente se define como una de las áreas prioritaria y se organizan los espacios de reflexión académica -a nivel gobierno- para dotar de herramientas que permitan el desarrollo de un enfoque de política exterior (geopolítico-comercial) que incorpore la posición país en este campo, se podría incidir sistemáticamente en otros temas sobre los cuales Costa Rica no ha logrado convencimiento en el subsistema Centroamérica. Adicionalmente, es preciso recordar 
que generar conocimiento sobre sostenibilidad ambiental no solo es una necesidad de actualidad, sino una oportunidad para que Costa Rica, que tiene la capacidad interna y algún nivel de legitimidad internacional para hablar de ambiente, comience a ejecutarlo. En este sentido, las posibilidades para posicionarse como líder en ambiente pueden llegar a ser amplias.

Una hoja de ruta inicial en la estrategia de internacionalización del conocimiento debe dar cuenta de algunos de los elementos que han sido presentados en este trabajo:

1. Es claro que pese a que las universidades públicas costarricenses cuentan con un alto nivel de prestigio en el subsistema Centroamérica, lo cierto del caso es que, cuando se habla de Relaciones Internacionales, la oferta costarricense es esencialmente privada, lo que implica, por un lado, para la Universidad Nacional de Costa Rica, una labor de liderazgo en el desarrollo de la iniciativa y, además, de una estrategia de Cancillería para acercar a actores públicos y privados en una estrategia ganar - ganar sin que existan roces de proceso.

2. Deben ser resueltas las diferencias de enfoque desde las cuales se enseñan Relaciones Internacionales y la perspectiva de lo que se espera de ellas por parte de la Cancillería. En este sentido, resulta sumamente esperanzador el reciente convenio entre la Escuela de Relaciones Internacionales de la UNA y este ente de Gobierno Central. Ahora bien, esto necesariamente pasa por la adquisición de modalidades académicas de formación de mayor flexibilidad, de una gran cercanía con los actores que se encuentran en la arena de la acción de la política exterior. En este sentido, tanto las universidades públicas y como las privadas deben iniciar un proceso de revisión que permita dimensionar, de mejor forma, las posibilidades de aplicación práctica de conocimientos.

3. Pese a que la academia de Relaciones Internacionales tiene poca o ninguna experiencia en la generación de Think Thank, se considera que estos sí pueden asumir el reto de asesorar la forma en que el conocimiento nacional debe proyectarse internacionalmente. Asimismo, no solo es una posibilidad de posicionar su propio conocimiento como academia, sino de guiar el proceso de internacionalización del conocimiento producido en otras ramas académicas, las cuales también pueden estar desarrollando temas de interés para la política exterior costarricense. Resulta interesarse preguntarse: ¿Por qué, además de impulsar la coordinación gobierno-academia, no se impulsa, a su vez, la coordinación interdisciplinaria? De esta manera, la oferta de conocimiento nacional sería más integral y exploraría nuevos nichos de conocimiento.

Para ello, se requiere mucho feed back de los actores que se encuentran en la administración de los grandes temas país. Se piensa también, al respecto, que la existencia de una gran oferta privada de educación superior en este campo puede ser también una ventana de oportunidad para ello, en la medida en que, teóricamente, estas casas de estudio podrían expresar los intereses de sectores puntuales en el escenario político nacional y, así, generar estrategias que faciliten arreglos o acuerdos país entre las élites. 
4. El tema de medio ambiente y sostenibilidad se ajusta a la imagen país que se quiere proyectar a través de la marca Costa Rica Esencial: básicamente, la de un país verde y garante de sostenibilidad. Entonces, ¿por qué no involucrar a la academia y al sector experto en la proyección de la imagen país? ¿Por qué no percibir esta estrategia con gran expectativa y convertirla en una aliada de la política comercial? ¿Por qué no migrar del soft power hacia el smart power?

¿Cuál es la razón de mantener a las universidades produciendo conocimiento, si este no se traduce en proyectos concretos que contribuyan al desarrollo del país? ¿Debemos replantearnos el rol que juegan las universidades en el desarrollo del país, más allá de la idea de graduar estudiantado? Las estadísticas apuntan que gobierno y academia sí desean trabajar coordinadamente. No obstante, falta mayor diálogo, comunicación asertiva y capacidad de negociación y planificación entre ambas esferas.

5. Para que la academia de Relaciones Internacionales costarricenses sea un agente en capacidad de generar sinergias y ganar legitimidad tanto a lo interno del proceso como en la generación de nuevos inputs y outputs en el subsistema región, se deben mejorar los criterios de pertinencia y relevancia de sus procesos de investigación. Esto pasa por dar un salto cualitativo a la investigación aplicada. Para ello debe vencerse el miedo al acercamiento entre los agentes Cancillería y academia, lo cual se logra mediante el desarrollo de experiencias aplicadas. En este sentido, se considera que la incorporación del Instituto en procesos compartidos de investigación puede ser la punta del iceberg para el avance. En una eventual estrategia de acercamiento pareciera claro que es la Universidad Nacional el primer aliado potencial con el que la Cancillería debería contar.

6. El posicionamiento internacional del conocimiento, además del trasfondo comercial, conllevaría una oportunidad para que Costa Rica lidere procesos de cooperación internacional. De esta manera, puede enriquecer, por ejemplo, el Catálogo de oferta de cooperación técnica, para la cooperación sur-sur y triangular del Ministerio de Planificación Económica (MIDEPLAN), en el cual los temas ambientales salen a la lustre: desarrollo sostenible y población ocupan el segundo lugar de la oferta y medio ambiente, el octavo lugar (MIDEPLAN, 2010). Ello podría resultar estratégico, en los esfuerzos de COMEX y Cancillería, para destacar el papel de Costa Rica en la OCDE y sus capacidades como oferente de cooperación. Debe tomarse en cuenta, también, según González M. (2014) cómo se debe institucionalizar la cooperación, con el fin de generar una agencia de cooperación que se convierta en un brazo estratégico de la cooperación hacia la triangulación y la sur-sur.

7. Tomando en cuenta la migración hacia las sociedades del conocimiento, resulta crucial sacar provecho del capital humano-intelectual, estructural y relacional con el que cuenta el país en temas de informáticas y comunicación. Se recomienda explotar el uso de plataformas informáticas y de telecomunicaciones, así como la interacción estratégica de las páginas web de uni- 
versidades y gobierno para proyectar los avances, iniciativas, enlaces estratégicos y productos resultantes del proceso de internacionalización del conocimiento. Para ello, el establecimiento de una línea discursiva base será esencial.

8. Finalmente, como parte de planificar el éxito del proceso, resulta necesario generar una estrategia a largo plazo, con la capacidad de prospectar escenarios, crear indicadores de análisis y un método adecuado de planificación y evaluación del proyecto. Este proyecto debe ser tema país, para que no sea vulnerable al vaivén de los cambios de administración. Se trata de crear una relación de reciprocidad entre gobierno y sector académico, instancias que, por años, se han mantenido tan distantes.

\section{Referencias}

Alpízar, M. \& Salas, L. (2015). Exploración del pensamiento costarricense en Relaciones Internacionales a la luz de las instituciones de educación superior que ofrecen la carrera. Práctica profesional. FOCAIS, Universidad Nacional de Costa Rica.

Bueno, E. (2000). El capital intelectual de la pyme: Una necesidad, un reto. España: Euroforum.

Bueno, E. Rodríguez, P. Salmador, M. (1999). Experiencias en medición del capital intelectual en España: El modelo intelect. España: Euroforum.

Dhanapala, J. (2011). Soft power, hard power and sustainable smart power. Recuperado de http:// transcurrents.com/tc/2011/02/soft power hard power and sust.html.

Donahue, J. \& Nye, J. (2000).Governance in a globalizing world. Washington DC: Brookings Institution.

González, M. (17 de octubre de 2014). Desafíos de la política exterior costarricense: Hacia una consolidación de política de Estado. Presentación del Ministro de Relaciones Exteriores y Culto, Manuel A. González Sáenz en la Universidad de Costa Rica. Ministerio de Relaciones Exteriores y Culto.

Kruger, K. (2006) El concepto de sociedad del conocimiento. Revista Bibliografía de Geografía y Ciencias Sociales. Universidad de Barcelona. Recuperado de http://www.ub.edu/geocrit/ b3w-683.htm

Méndez, M. (2012). ¿Poder inteligente? La doctrina Obama y Guantánamo. Universidad Nacional de Costa Rica. Recuperado de http://www.alainet.org/images/Poderlnteligente.pdf 
MIDEPLAN. (2010). Catálogo de Oferta de la Cooperación Técnica de Costa Rica. Ministerio de Planificación Económica y Política Comercial. Recuperado de https://documentos. mideplan.go.cr/alfresco/d/d/workspace/SpacesStore/d413032b-30b5-4ce4-a5ebad101c140516/Catalogo-oferta-cooperac-tec-CR-978-9977-73-042-4.pdf?guest=true

Montobbio, M. (2013). Geopolítica del pensamiento.Barcelona: Real Instituto el Cato. Centre for Internacional Affairs.

Peluffo, B. \& Catalán, E. (2002). Introducción a la Gestión del Conocimiento y su aplicación al Sector Público. CEPAL, Santiago de Chile. Recuperado de: http://archivo.cepal.org/ pdfs/2002/S2002617.pdf

Ranking Web de Universidades. (2014). América Central y el Caribe. Recuperado de http://www. webometrics.info/es/america central caribe.

UNESCO. (2005). Hacia las sociedades del conocimiento. Organización de las Naciones Unidas para la Educación, la Ciencia y la Cultura. Recuperado de: http://unesdoc.unesco.org/images/0014/001419/141908s.PDF.

Vigésimo Informe del Estado de la Nación (2015). Continuidades y cambios: De la administración Chinchilla Miranda a la administración Solís Rivera. La política exterior de Costa Rica (20142015). Estado de la Nación. Recuperado de http://estadonacion.or.cr/files/biblioteca virtual/021/politica/Escuela de Relaciones Internacionales.pdf. 